\title{
The Influence of Malassez Epithelial Residue in Inflammatory Periodontal Tissues on the Number of Periodontal Stem Cells and Their Differentiation Ability
}

\author{
Hua Fu* Xiaodong Hu, Yanfeng Xia \\ Department of Stomatology, Shangyu People's Hospital, Shaoxin, China \\ Email address: \\ crest0932@163.com (Hua Fu),7408419@qq.com (Xiaodong Hu),2921418113@qq.com (Yanfeng Xia) \\ ${ }^{*}$ Corresponding author
}

\section{To cite this article:}

Hua Fu, Xiaodong Hu, Yanfeng Xia. The Influence of Malassez Epithelial Residue in Inflammatory Periodontal Tissues on the Number of Periodontal Stem Cells and Their Differentiation Ability. Science Discovery. Vol. 7, No. 5, 2019, pp. 318-322. doi: 10.11648/j.sd.20190705.20

Received: September 10, 2019; Accepted: November 12, 2019; Published: November 13, 2019

\begin{abstract}
To investigate the effect of Malassez epithelial surplus in inflammatory periodontal tissues on the number and differentiation ability of periodontal stem cells. 30 patients with periodontitis admitted to the hospital from November 2014 to April 2017 were selected as the observation group. A total of 30 healthy oral examination patients were selected as control group. Periodontal tissue in the 2 groups were taken and the periodontal ligament cells, the complete inflammatory periodontal ligament stem cells by using the low density screening, purification, and periodontal tissue were obtained by using differential digestion method of Malassez epithelial remaining, were observed under inverted microscope in the periodontal tissue Malassez epithelial residual form, will be in the periodontal tissue Malassez epithelial surplus together with inflammatory periodontal ligament stem cells, RT-PCR technique has been applied to the determination of ALP and Runx-2 mRNA expression level to observe the differentiation ability. Flow cytometry showed that the cell markers CD29 and CD90 in the cultured periodontal stem cells were positive $(88.9 \%$ and $99.7 \%$, respectively). A hematopoietic stem cell marker CD45 was negative $(0.3 \%)$. The inverted microscope results showed that the residual epithelial cells of Malassez, isolated and cultured, had a large nuclear to cytoplasmic ratio, and the different cells were arranged relatively closely, with the overall cell structure of "paving stone". The expression levels of ALP and runx-2 mRNA in the observation group were higher than those in the control group $(\mathrm{P}<0.05)$. Malassez epithelial surplus in inflammatory periodontal tissues can promote the proliferation of periodontal stem cells and improve the ability of osteogenic differentiation.
\end{abstract}

Keywords: Inflammatory Periodontal Tissue, Malassez Residual Epithelium, Periodontal Stem Cells, Proliferation Capacity, Differentiation Ability

\section{炎症牙周组织中Malassez上皮剩余对牙周干细胞数量及其分化能 力影响}

付华, 胡晓东, 夏岩锋

绍兴市上虞人民医院口腔科, 绍兴, 中国

邮箱

crest0932@163.com (付华),7408419@qq.com(胡晓东),2921418113@qq.com (夏岩锋)

摘要: 目的: 探讨炎症牙周组织中Malassez上皮剩余对牙周干细胞数量及其分化能力的影响。方法: 取 2014 年11月-2017 年4月医院收治的牙周炎患者 30 例, 设为观察组; 取同期入院健康口腔检查者 30 例, 设为对照组。分别取2组牙周组 
织并获得牙周膜细胞, 利用低密度法完成炎症牙周膜干细胞的篮选、纯化, 利用差速消化法获得牙周组织中 Malassez 上皮剩余, 倒置显微镜下观察牙周组织中Malassez上皮剩余形态, 将牙周组织中Malassez上皮剩余与炎症牙周膜干细 胞进行共同培养, 利用RT-PCR技术测定ALP及Runx-2 mRNA表达水平观察其分化能力。结果: 流式细胞仪测定结果 显示：体外分离培养的牙周干细胞中细胞标志物CD29、CD90呈阳性，分别为 $88.9 \%$ 与 $99.7 \%$; 而造血干细胞相关标 记物CD 45 为阴性, 为 $0.3 \%$; 倒置显微镜结果显示: 分离培养的Malassez上皮剩余细胞细胞有较大的核质比, 并且不 同的细胞之间排列相对紧密, 细胞整体呈“铺路石”样结构; 观察组牙周组织共同培养后ALP及Runx-2 mRNA表达水 平, 均高于对照组 $(\mathrm{P}<0.05)$ 。结论：炎症牙周组织中Malassez上皮剩余能促进牙周干细胞增殖能力, 利用提高成 骨分化能力。

关键词: 炎症牙周组织, Malassez上皮剩余, 牙周干细胞, 增殖能力, 分化能力

\section{1. 引言}

牙周支持组织包括两种软组织 (牙龁、牙周膜) 与 两种硬组织 (牙槽骨、牙骨质) [1]。从人体解剖学角度 来说 [2], 牙龈组织覆盖牙颈部周围、牙槽突表达的口腔 黏膜及上皮、下方结缔组织; 而牙周膜则连接牙齿与牙 槽骨致密结缔组织, 在牙齿稳固、功能中发挥了重要的 作用。文献报道显示[3]: 牙周膜组织中存在尚未分化的、 具备自我更新、多向分化潜能的细胞, 临床将其称为牙 周膜干细胞 (PDLSCs), 是牙周支持组织再生、重建 的基础。研究表明 [4-5]: PDLSCs是一种牙周组织再生 公认的细胞, 有助于促进干细胞的增殖、分化。但是, 炎症微环境下由于炎症因子的刺激等, 会造成PDLSCs 细胞的分化能力下降, 在一定程度上影响成骨能力, 不 利于牙周组织的再生。Malassez上皮剩余 (ERM) 是牙 根发育过程中Hertwig' $\mathrm{s}$ 上皮根鞘 (HERS) 退化过程中 的细胞结构, 被视为无用的组织。研究表明 [6-7]: 从正 常牙周组织中分离的PDLSCs通过与 HERS共同培养能 提高牙周干细胞数量及分化能力, 但是该结论有待验证。 为了探讨炎症牙周组织中Malassez上皮剩余对牙周干细 胞数量及其分化能力的影响。取 2014年11月-2017年4月 医院收治的牙周炎患者 30 例及健康口腔体检者 30 例, 报 道如下。

\section{2. 资料与方法}

\section{1. 临床资料}

取 2014年11月-2017年4月医院收治的牙周炎患者30 例, 设为观察组, 男18例, 女12例, 年龄 (16-45) 岁, 平均 $(32.84 \pm 4.82)$ 岁, 病程 (1-2) 年, 平均 $(1.02 \pm 0.35)$ 年; 纳入标准: (1) 符合牙周炎临床诊断标准者; (2) 均经过生化指标、口腔影像学检查最终得到确诊; (3) 均自愿取牙周组织。取同期入院健康口腔检查者 30 例, 设 为对照组, 男17例, 女13例, 年龄 (17-47) 岁, 平均 (33.04 \pm 4.83$)$ 岁。排除标准: (1) 不符合牙周炎临床诊 断及纳入标准者; (2) 资料不全或难以配合诊断者; (3) 合并严重心、肝、肾功能异常及肝功能异常者。本课题在 伦理委员会批准、监督下进行, 患者对治疗方法等知情同 意。

\section{2. 主要仪器和试剂}

为了保证试验的顺利完成, 采用的主要仪器和试剂主 要由YJ-875型超净工作台、上皮培养基、茜素红、胎牛 血清（FBS）、超声震荡仪、 $50 \mathrm{~cm} 2$ 培养瓶、6 孔培养板、 12 孔培养板、倒置相差显微镜及照相系统、ALP 检测试 剂盒等, 相关试剂和仪器厂家如下, 见表1。

表1 主要仪器和试剂。

\begin{tabular}{ll}
\hline 主要仪器和试剂 & 厂家/批号 \\
\hline YJ-875型超净工作台 & 苏州净化设备厂 \\
上皮培养基 & sciencell, 美国 \\
胎牛血清 (FBS) & 杭州四季青公司 \\
超声震荡仪 & 上海生源器械厂 \\
$50 \mathrm{~cm}^{2}$ 培养瓶、6 孔培养板、12 孔培养板 & Falcon, 美国 \\
倒置相差显微镜及照相系统 & Olympus, 日本 \\
ALP 检测试剂盒 & Takara日本 \\
\hline
\end{tabular}

注: 研究中所使用的仪器和试剂均由相应公司提供。

\section{3. 方法}

分别取2组牙周组织并获得牙周膜细胞, 利用低密度 法完成牙周膜干细胞的笁选、纯化, 利用差速消化法获得 牙周组织中Malassez上皮剩余, 倒置显微镜下观察牙周组 织中Malassez上皮剩余形态, 将牙周组织中Malassez上皮 剩余与炎症牙周膜干细胞进行共同培养, 利用RT-PCR技 术测定ALP及Runx-2 mRNA表达水平。

(1) 牙周膜干细胞的篎选、纯化。分别取 2 组牙周组织, 将其放置在超净工作台上, 采用组织自块酶消化 法向组织中加入 $150 \mathrm{~mL} / \mathrm{L}$ FBS的a-MEM培养基进 行培养, 待细胞融合 $80.0 \%$ 后进行传代培养。利用 低密度法完成牙周干细胞的篮选、纯化, 传代培 养，取第四代细胞备用，利用差速消化法获得牙 周组织中Malassez上皮剩余, 倒置显微镜下观察 细胞形态 [8]。

（2）牙周膜干细胞鉴定。取第4代牙周膜干细胞利用胰 酶消化法制备单细胞悬液, 将细胞密度调节为 $1 \times 10^{6} / 200 \mathrm{uL}$, 分别将其放置在EP管中, PBS下进 行3次清洗, 向管中加入 $2 \mathrm{uL}$ 鼠抗人CD29、CD90 抗体及 $2 \mathrm{uL}$ 兔抗人 CD 45 抗体, $4{ }^{\circ} \mathrm{C}$ 下进行 $1 \mathrm{~h}$ 捊育, 利用流式细胞仪完成各标记物鉴定[9]。 
(3) Malassez上皮剩余与炎症牙周膜干细胞共培养。 将 ERM 以 $5.5 \times 10^{3} / \mathrm{cm}^{2}$ 接种在 $0.4 \mathrm{um}$ 的 Transwell 小室上层, 加入培养液并放入培养箱中进行 $24 \mathrm{~h}$ 培养，将第 4 代牙周干细胞，加入 $10 \mathrm{~mL} / \mathrm{L}$ 培养 基, 加入培养箱中, 待细胞对数生长后完成 Malassez上皮剩余与炎症牙周膜干细胞共培养 [10]。

(4) ALP及Runx-2 mRNA表达水平测定。利用RT-PCR 技术测定ALP及Runx-2 mRNA表达水平, 以 cDNA 作为模板, 以GAPDH为内参照, 有关操作严格遵 循Takara公司生产的试剂盒步骤完成, 利用荧光 检测仪完成ALP及Runx-2 mRNA表达水平测定 [11]。

\section{4. 统计分析}

采用SPSS18.0软件处理, 计数资料行 $x^{2}$ 检验, 采用 $\mathrm{n}$ $(\%)$ 表示, 计量资料行 $\mathrm{t}$ 检验, 采用 $(\bar{x} \pm s)$ 表示, $\mathrm{P}<0.05$ 差异有统计学意义。

\section{3. 结果}

\section{1. 分离牙周干细胞的鉴定}

流式细胞仪测定结果显示: 体外分离培养的牙周干细 胞中细胞标志物 CD29、CD90呈阳性[12], 分别为 $88.9 \%$ 与 $99.7 \%$; 而造血干细胞相关标记物CD 45 为阴性, 为 $0.3 \%$, 由此看出: 分离的干细胞为牙周干细胞, 见图1。
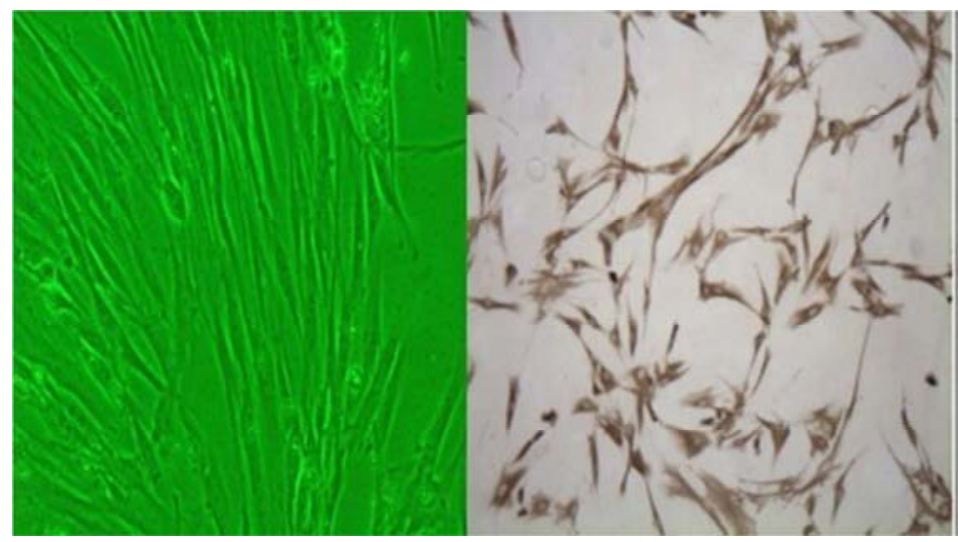

图1 分离牙周干细胞的鉴定。

\subsection{Malassez上皮剩余细胞形态}

倒置显微镜结果显示：分离培养的Malassez上皮剩余 细胞细胞有较大的核质比, 并且不同的细胞之间排列相对 紧密, 细胞整体呈“铺路石”样结构[13], 见图2。

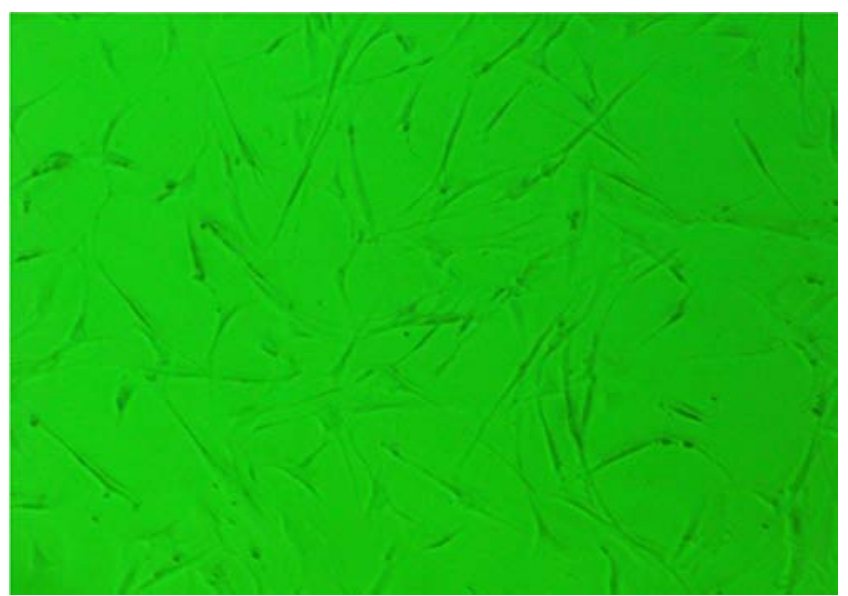

图2 Malassez上皮剩余细胞形态（×40）。

\section{3. 两组不同牙周组织共培养后ALP及Runx-2 mRNA表 达水平比较}

观察组牙周组织共同培养后ALP及Runx-2 mRNA表 达水平，见表2。
表2 2组不同牙周组织共培养后ALP及 Runx-2 mRNA表达水平比较 $(\bar{x} \pm s)$ 。

\begin{tabular}{llll}
\hline 组别 & 例数 & ALP & Runx-2 mRNA \\
\hline 观察组 & 30 & $0.86 \pm 0.12$ & $1.02 \pm 0.21$ \\
对照组 & 30 & $0.05 \pm 0.02$ & $0.31 \pm 0.12$ \\
$\mathrm{t}$ & $/$ & 6.793 & 5.571 \\
$\mathrm{P}$ & $/$ & $<0.05$ & $<0.05$ \\
\hline
\end{tabular}

\section{4. 结论}

本研究中, 流式细胞仪测定结果显示: 体外分离培养 的牙周膜干细胞中细胞标志物CD29、CD90呈阳性, 分别 为 $88.9 \%$ 与 $99.7 \%$; 而造血干细胞相关标记物CD45为阴性, 为 $0.3 \%$, 由此看出: 分离的干细胞为牙周膜干细胞。本研 究中, 观察组牙周组织共同培养后ALP及Runx-2 mRNA表 达水平, 均高于对照组 $(\mathrm{P}<0.05)$ 。由此看出：炎症牙周 组织中 Malassez上皮剩余连续培养有助于提高ALP及 Runx-2 mRNA表达水平。炎症牙周组织中Malassez上皮剩 余能提高成骨诱导分化能力, 可能提高临床修复效果 [14], 但尚需要进一步验证和研究。

\section{5. 讨论}

牙龈组织是指覆盖在牙槽突表面和牙颈部周围口腔 黏膜上皮及下方的结缔组织, 属于牙周组织的重要组成 
部位。正常牙龈组织色粉红, 表明存在点彩, 而在炎症 状态下牙龈组织将会引起充血肿胀, 造成表面点彩消失 [15]。文献报道显示[16]: 牙周炎是累及不同牙周支持组 织的慢性感染性疾病, 是引起患者失牙的重要原因。对 于重度牙周病患者由于牙周支持组织出现不可逆丧失, 导致牙齿发生松动、移位, 影响牙齿的稳固和健康。文 献报道显示: 微环境对于干细胞的自我增殖、分化等具 有一定的影响, 并且在炎症、衰老等情况下能降低牙周 干细胞多向分化能力, 从而造成干细胞成骨分化能力降 低, 能直接对牙周组织的再生产生明显的影响。近年来, 组织工程在牙周缺损组织修复中得到应用, 而种子细胞 则是修复的关键内容。牙周干细胞是一种特殊的细胞, 本研究中, 倒置显微镜结果显示: 分离培养的Malassez 上皮剩余细胞细胞有较大的核质比, 并且不同的细胞之 间排列相对紧密, 细胞整体呈“铺路石”样结构。该细胞 具有高度增殖、分化能力, 并且能在特定的作用下向成 骨、成脂方向分化, 并且该类细胞经过诱导后能形成牙 骨质/牙周膜样结构。因此, 本文通过研究牙周干细胞能 为临床牙周疾病的治疗提供依据和参考。Malassez上皮剩 余（ERM）是牙根发育时期Hertwig's上皮根鞘退化后形 成的上皮结构组织。文献报道显示: ERM可以表达分泌 多种细胞角蛋白、胞外机制、细胞表面蛋白及生长因子 等。同时, ERM还可以合成一些与间充质细胞关系更加 密切的功能蛋白, 包括: 骨桥蛋白OPN、骨唾液蛋白、 骨保护素OPG及金属蛋白酶MMP-13级金属蛋白酶组织 抑制剂、成釉蛋白等 $[17,18]$, 在矿化组织形成、牙周组 织的改建中发挥了重要的作用。文献报道显示: 将牙周 膜干细胞与上皮根鞘共同培养时, 上皮根鞘能发挥良好 的作用, 有助于诱导牙周膜干细胞向牙骨质细胞方向分 化, 由此看出: 炎症牙周组织中Malassez上皮剩余在牙根 /牙周发育过程中能引起上皮间充质而发生交互作用, 从 而能提高成骨细胞的分化能力 $[19,20]$ 。

国外学者研究表明: 在牙齿发生移动时张力区的 ERM细胞将会增多, 呈较强增殖和分化活性, 在牙齿的 移动中发挥了重要的作用[21]。同时, ERM还能表达骨 保护素OPG, 可以抑制骨细胞的分化活性。ERM在感受 外界的炎症和肿瘤条件的刺激后可以表现出极强的增殖 和分化能力, 具有强大的分化潜能。能分泌生长因子并 经transwell小室滤膜等作用于牙周膜干细胞中, 从而能提 高成骨分化能力 [22]。Farea用人脱落乳牙与ERM共同培 养发现, ERM能协同转化生长因子TGF- $\beta 1$ 的增殖, 能诱 导乳牙的成牙骨质细胞分化, 而这种活性通过高碱性磷 酸酶ALP活性及其矿物的沉积和蛋白质表达的上调来实 现[23]。国内学者研究表明: 将牙周膜干细胞与上皮根鞘 进行间接共同培养时, 上皮根鞘能有效的诱导牙周膜干 细胞向成牙骨质细胞方向分化。由此看出: ERM能提高 ALP及Runx-2 mRNA表达水平, 有助于显示较强的分化 能力, 该结论与经典的牙根重组实验结果相仿。综上所 述, 炎症牙周组织中Malassez上皮剩余能促进牙周干细胞 增殖能力, 利用提高成骨分化能力, 能为临床牙周炎治 疗提供新的思路。

\section{参考文献}

[1] Andreas N, Carl-Peter C, Michael R, et al. The Comprehensive AOCMF Classification System: Condylar Process Fractures-Level 3 Tutorial [J]. Craniomaxill of acial Trauma \& Reconstruction, 2014, 7 (7): S044-S058.

[2] 聂嘉, 张博, 顾斌, 等.p38丝裂原活化蛋白激酶在炎症微 环境作用下对牙周膜干细胞成骨分化的影响 [J].中国医学 科学院学报, 2015, 37 (1):1-7.

[3] Martinez C, Rath S, Van Gulden S, et al. Periodontal ligament cells cultured under steady-flow environments demonstrate potential for use in heart valve tissue engineering. Tissue Eng Part A.2013; 19 (3/4):458-466.

[4] 李晓光, 王一珠, 郭斌. 慢性牙周炎中肿瘤坏死因子 $a$ 对骨 髓间充质干细胞成骨分化的调控作用 $[\mathrm{J}]$. 华西口腔医学杂 志, 2017，35 (3) :334-338.

[5] 王红, 陈琦, 刘文佳, 等.曲古抑菌素A对肿瘤坏死因子 $\alpha$ 诱导的炎症微环境下牙周膜干细胞成骨分化能力的影响 [J]. 中华口腔医学杂志, 2016, 51 (4) :235-241.

[6] Limjeerajarus Chalida Nakalekha, Osathanon Thanaphum, Manokawinchoke Jeeranan, et al. Iloprost up-regulates vascular endothelial growth factor expression in human dental pulp cells in vitro and enhances pulpal blood flow in vivo [J]. Journal of endodontics, 2014, 40 (7): 925-926.

[7] Andy H. Choi, Jukka Matinlinnal \& Besim ben-Nissan.Effects of micromovement on the changes in stress distribution of partially stabilized zirconia (PS-ZrO2) dental implants and bridge during clenching: A three-dimensional finite element analysis [J]. Acta Odontologica Scandinavica, 2013, 71 (82): 72-81.

[8] Cehreli ZC, Isbitiren B, Sara S, Erbas G. Regenerative endodontic treatment (revascularization) of immature necrotic molars medicated with calcium hydroxide: Acase series [J]. Journal of endodontics, 2015, 37 (9): 1327-1330.

[9] 薛范, 李蓓, 谈珺, 等。脂多糖诱导的内质网应激在牙周 膜干细胞中的表达及其对成骨分化的影响 [J]. 中华口腔医 学杂志, 2015,50（9）:548-553.

[10] Yoon TH, Madden JC, Chang WG. A technique to restore worn denture teeth on a partial removable dental prosthesis by using ceramic onlays with CAD/CAM technology. J Prosthet Dent. 2013; 110 (4): 331-332.

[11] Hyun Nam,Jaewon Kim, et al. Expression Profile of the Stem Cell Markers in Human Hertwig's Epithelial Root Sheath/Epithelial Rests of Malassez Cells. Molecules and Cells. 2011, April 30, 31, 355-360.

[12] 邵江红, 陈芳, 孔宇.miR-30a在雷帕霉素刺激人炎症牙周 膜干细胞后的表达情况及其与Beclin1基因的靶向调控作用 的研究 [J].口腔医学研究, 2017, 33 (6) :658-662.

[13] Awad D, Stawarczyk B, Liebermann A, et al.Translucency of esthetic dental restorative $\mathrm{CAD} / \mathrm{CAM}$ materials and composite resins with respect to thickness and surface roughness.J Prosthet Dent.2015, 113 (6) :534-540. 
[14] JIMIN XIONG, STAN GRONTHOS, et al. Role of the epithelial cell rests of Malassez in the development, maintenance and regeneration of periodontal ligament tissues. Periodontology, 2000, Vol, 63, 2013, 217-223.

[15] 刘娜, 石海刚, 张维, 等.炎症微环境作用下经典及非经典 Wnt通路平衡对牙周膜干细胞成骨分化的调控作用[J].中华 口腔医学杂志, 2016, 51 (11) :673-679.

[16] 徐孟丹, 周咏, 邹多宏.拉布拉多犬骨髓基质干细胞与牙周 干膜细胞裸鼠皮下成骨能力的比较性研究 [J].安徽医科大 学学报, 2016, 51 (9): 1244-1247.

[17] YUKA SHINMURA, SHUHEI TSUCHIYA, et al. Quiescent Epithelial Cell Rests of Malassez Can Differentiate Into Ameloblast-Like Cells. J. Cell. Physiol. 2008, 217: 728-738.

[18] Naohiko Hasegawa, Hiroyuki Kawaguchi, Tetsuji Ogawa, et al. Immunohistochemical characteristics of epithelial cell rests of Malassez during cementum repair. J Periodont Res 2003; 38 ; $51-56$.
[19] 王烟岗, 王萧萧, 陈天宇, 等. 牙周膜干细胞与牙周膜细 胞甲状旁腺激素受体表达的比较 [J]. 中国组织工程研究, 2015, 19 (10):1562-1569.

[20] N. Aida, T. Ushikubo, F. Kobayashi, et al, Actin stabilization induces apoptosis in culturedporcine epithelial cell rests of malasez. J International Endodontic, 2016, 49, 663-669.

[21] Yüksel Korkmaz, Franz-Josef Klinz, et al. The Ca2+-binding protein calretinin is selectively enriched in a subpopulation of the epithelial rests of Malassez. Cell Tissue Res, 2010, 342; 391-400.

[22] O. Tadokoro, T. Maeda, K. J. Heyeraas, et al, Merkel-like cells in Malassez epithelium in the periodontal ligament of cats: an immunohistochemical, confocal-laser scanning and immuno electron, microscopic investigation. J Periodont Res, $2002 ; 37 ; 456-463$.

[23] Farea M, Husein A, Halim AS, et al. Cementoblastic lineage formation in the cross-talk between stem cells of human exfoliated deciduous teeth and epithelial rests of Malassez cells. Clin Oral Investig,2016, 20. 